\title{
Eine Bemerkung zu Clustereigenschaften *
}

\author{
D. MaISON
}

Max-Planck-Institut für Physik und Astrophysik, München

Eingegangen am 13. Mai 1968

Abstract. It is proved that in a unitary representation of the Poincaré- or Galileigroup the infinitesimal translations have a spectral measure without singular continuous part.

Zur Beschreibung physikalischer Streuprozesse in einer lokalen Quantentheorie spielen bekanntlich die räumlichen Clustereigenschaften der Erwartungswerte von Produkten lokaler Observabler eine wesentliche Rolle [1]. DopLICHER et al. [2] haben gezeigt, daß für asymptotisch abelsche Systeme sich diese Clustereigenschaften aus der Annahme herleiten lassen, daß ein eindeutig bestimmter invarianter Zustandsvektor im Hilbertraum existiert (Vakuum). Eine lokale, relativistische Quantentheorie ist nun bezüglich raumartiger Translationen auf Grund der Lokalitätsbedingung schwach asymptotisch abelsch und besitzt somit räumliche Clustereigenschaften. Es erhebt sich die Frage, ob auch für zeitartige Translationen ähnliche Aussagen möglich sind. Diese Frage kann, wie der folgende Satz zeigt, für Poincaré- bzw. Galileiinvariante Theorien mit eindeutig bestimmtem Vakuum zumindest für spezielle Cluster bejaht werden, dabei spielt jedoch die lokale Struktur keine Rolle.

Satz 1. Seig $\rightarrow U_{g}$ eine unitäre Darstellung ${ }^{1}$ der Poincaré-(Galilei-)gruppe $\mathscr{G}$ im separablen Hilbertraum $\mathscr{H}, \mathfrak{A}$ eine $C^{*}$-Algebra von linearen Operatoren in $\mathscr{H}$, so da $\alpha_{g}(A):=U g A U_{g}^{-1}$ in $\mathfrak{A}$ liegt für alle $g \in \mathscr{G}$. Sei ferner $E_{0}$ die orthogonale Projektion auf den Teilraum der translationsinvarianten Vektoren in $\mathscr{H}$ und 2 ein beliebiger Einheitsvektor in $E_{4}$, dann gilt;

$$
\lim _{\lambda \rightarrow \infty}\left(\varphi, A \alpha_{\lambda e}(B) \varphi\right)=\left(\varphi, A E_{0} B \varphi\right) \quad \text { für } \quad \varphi \in E_{0} \mathscr{H}
$$

und somit

$$
\lim _{\lambda \rightarrow \infty}\left(\Omega, A \alpha_{\lambda e}(B) \Omega\right)=(\Omega, A \Omega)(\Omega, B \Omega)
$$

falls $\Omega$ der einzige translationsinvariante Vektor in $\mathscr{H}$ ist.

* Nachdem das vorliegende Manuskript bereits im Druck war, fand der Autor eine Bemerkung von H. Araki (Progr. theor. Phys. 32, 844 (1964)) mit derselben Aussage (ohne Beweis).

${ }^{1}$ Wir betrachten einfachheitshalber nur ",physikalische" Darstellungen mit nichtnegativer Masse und diskretem Spin; der Satz gilt jedoch für alle unitären Darstellungen. 
Beweis. Der Beweis beruht auf der Totalstetigkeit des Spektralmaßes der infinitesimalen Translationen $P_{\mu}$ bezüglich des Lebesguemaßes, wenn man sich auf $\left(1-E_{0}\right) \mathscr{H}$ beschränkt. Bekanntlich hat $P_{\mu}$ in einer Poincaré-(Galilei-)invarianten Theorie keine Eigenwerte außer $P_{\mu}=0$. Das Spektralmaß könnte jedoch einen stetigen, nicht totalstetigen Anteil auf dem kontinuierlichen Spektrum enthalten. Der folgende Satz 2 zeigt, daß dies ausgeschlossen werden kann.

Sei also $\varphi \in E_{0} \mathscr{H}, B, A \in \mathfrak{A}$ und $F(p)$ die Spektralschar von $e \cdot P$ $=e^{\mu} P_{\mu}$, dann gilt

$$
\begin{aligned}
\left(\varphi, A \alpha_{\lambda e}(B) \varphi\right)= & \left(\varphi, A e^{i \lambda e \cdot P} B e^{-i \lambda e \cdot P} \varphi\right) \\
= & \int e^{i \lambda p}(\varphi, A d F(p) B \varphi)=\left(\varphi, A E_{0} B \varphi\right) \\
& +\int e^{i \lambda p} \frac{d\left(\varphi, A\left(1-E_{0}\right) F(p) B \varphi\right)}{d p} d p .
\end{aligned}
$$

Aus der absoluten Stetigkeit von $\left(1-E_{0}\right) F(p)$ folgt

$$
\frac{d\left(\varphi, A\left(1-E_{0}\right) F(p) B \varphi\right)}{d p} \in \mathscr{L}_{1}(d p)
$$

und mit dem Riemann-Lebesgue-Lemma die Behauptung.

Corollar. Gilt zusätzlich zu den Voraussetzungen von Satz 1 noch $\overline{\mathfrak{Q} E_{0} \mathscr{H}}=\mathscr{H}$ (z. B. $E_{0}=P_{\Omega}$ mit $\Omega$ zyklisch), so folgt $\lim _{\lambda \rightarrow \infty}\left(\chi_{1}, U_{\lambda_{e}}, \chi_{2}\right)$ $=\left(\chi_{1}, E_{0} \chi_{2}\right)$ für alle $\chi_{i} \in \mathscr{H}(i=1,2)$.

Bemerkung. Seien $\chi_{1}, \chi_{2}$ Wellenpakete, die Zuständen lokalisierter Teilchen entsprechen

$$
\left|\chi_{i}\right\rangle=\int f_{i}(\boldsymbol{k})\left|\boldsymbol{k}, m_{i}\right\rangle \frac{d^{3} k}{\sqrt{\boldsymbol{k}^{2}+m_{i}^{2}}} \operatorname{mit} f_{i}(\boldsymbol{k}) \in \mathscr{L}^{2}\left(R^{3}\right)
$$

dann folgt aus dem Corollar $\lim _{t \rightarrow \infty}\left(\chi_{1}, U_{t} \chi_{2}\right)=0$, was die anschauliche Erwartung rechtfertigt, daß das Wellenpaket im Laufe der Zeit zerfließt.

Satz 2. Sei $U_{g}$ eine unitäre Darstellung der Poincaré-(Galilei-)gruppe im separablen Hilbertraum $\mathscr{H}^{2}, E_{0}$ die orthogonale Projektion auf den Teilraum der translationsinvarianten Vektoren in $\mathscr{H}, e$ ein beliebiger Einheitsvektor in $E_{4}$ und $E($.$) das Spektralma \beta$ von $e \cdot P$, so ist $\left(1-E_{0}\right) E($. totalstetig bezüglich des Lebesguemaßes; das bedeutet u. a., daß die zugehörige Spektralschar $\left(1-E_{0}\right) F(p)=\left(1-E_{0}\right) E([-\infty, p])$ absolut stetig ist.

Beweis. Wir führen den Beweis nur für die Poincarégruppe; der für die Galileigruppe verläuft ganz analog (an Stelle der Bahn $p^{2}=m^{2}$ tritt $\boldsymbol{p}^{2}+$ const. $\left.=2 m p_{0}\right)$.

Wir zerlegen die Darstellung $U_{g}$ in irreduzible Darstellungen [3]; sei $\mathscr{H}=\int \bigoplus \mathscr{H}_{m, s} d \varrho\left(m^{2}\right) \oplus E_{0} \mathscr{H}$ die entsprechende Zerlegung von $\mathscr{H}$.

${ }^{2}$ Siehe Fußnote ${ }^{1}$.

4 Commun. math. Phys., Vol. 10 
Das Skalarprodukt in $\mathscr{H}_{m, s}$ lautet

$$
\left(\Phi_{m, s}, \Psi_{m, s}\right)_{m, s}=\sum_{l=-s}^{s} \int_{V_{+}} \Phi_{m, s}^{*}(p, l) \Psi_{m, s}(p, l) \delta\left(p^{2}-m^{2}\right) d^{4} p
$$

Wir unterscheiden drei Fälle, je nachdem ob $e$ zeitartig, raumartig oder lichtartig ist:

1. $e^{2}=1$.

Sei O.E. $\quad e=(1,0,0,0)$, dann ist $e \cdot P=P_{0}$.

Führen wir für $\boldsymbol{p}$ Polarkoordinaten ein, so wird

$$
\delta\left(p^{2}-m^{2}\right) d^{4} p=\frac{1}{2} \sqrt{p_{0}^{2}-m^{2}} \delta\left(|\boldsymbol{p}|-\sqrt{p_{0}^{2}-m^{2}}\right) d p_{0} d|\boldsymbol{p}| d \Omega(\boldsymbol{p}) .
$$

2. $e^{2}=-1$.

Sei O.E. $\quad e=(0,0,0,1)$, dann ist $e \cdot P=P_{3}$.

Wir setzen $p_{0}=r \cos h \alpha, p_{1}=r \sin h \alpha \cos \beta, p_{2}=r \sin h \alpha \cdot \sin \beta$ dann gilt $\delta\left(p^{2}-m^{2}\right) d^{4} p=\frac{1}{2} \sqrt{p_{3}^{2}+m^{2}} \delta\left(r-\sqrt{p_{3}^{2}+m^{2}}\right) \sin h \alpha \Theta(\alpha) d p_{3} d r d \alpha d \beta$.

3. $e^{2}=0$.

Sei O.E. $\quad e=\frac{1}{\sqrt{2}}(1,0,0,1)$, dann ist $e \cdot P=\frac{P_{0}-P_{3}}{\sqrt{2}}$.

Wir setzen

dann gilt

$$
\xi=\frac{p_{0}-p_{3}}{\sqrt{2}}, \quad \eta=\frac{p_{0}+p_{3}}{\sqrt{2}}, \quad p_{1}=r \cos \alpha, p_{2}=r \sin \alpha
$$

$$
\delta\left(p^{2}-m^{2}\right) d^{4} p=\delta\left(r-\sqrt{2 \xi \eta-m^{2}}\right) d \xi d \eta d r d \alpha .
$$

Sei $E_{m, s}($.$) die Komponente des Spektralmaßes von \ell \cdot P$ in $\mathscr{H}_{m, s}$ und $N$ eine Lebesgue-Nullmenge in $R^{1}$, so gilt

1. Fall

$$
\begin{gathered}
\left(\Phi_{m, s}, E_{m, s}(N) \Phi_{m, s}\right)_{m, s}=\sum_{l=-s}^{s} \int_{V+\cap}\left(\int_{N_{p_{0}}}\left|\bar{\Phi}_{m, s}\left(p_{0}, \frac{\boldsymbol{p}}{|\boldsymbol{p}|}, l\right)\right|^{2} \sqrt{p_{0}^{2}-m^{2}} d p_{0}\right) \\
\cdot d \Omega=0 .
\end{gathered}
$$

2. Fall

$$
\begin{aligned}
\left(\Phi_{m, s}, E_{m, s}(N) \Phi_{m, s}\right)_{m, s}= & \left.\sum_{l=-8}^{s} \int_{V_{+}} \bigcap_{N_{p_{3}}}\left|\Phi_{m, s}\left(p_{3}, \alpha, \beta, l\right)\right|^{2} \sqrt{p_{3}^{2}+m^{2}} d p_{3}\right) \\
& \cdot \sin h \alpha \Theta(\alpha) d \alpha d \beta=0 .
\end{aligned}
$$

3. Fall

$$
\left(\Phi_{m, s}, E_{m, s}(N) \Phi_{m, s}\right)_{m, s}=\sum_{l=-s}^{s} \int_{V_{+} \cap N \xi}\left(\int_{N \xi}\left|\Phi_{m, s}(\xi, \eta, \alpha, l)\right|^{2} d \xi\right) d \eta d \alpha=0
$$

wobei wir

gesetzt haben.

$$
\left.\Phi_{m, s}(p, l)\right|_{p^{2}=m^{2}}=\left\{\begin{array}{l}
\bar{\Phi}_{m, s}\left(p_{0}, \frac{\boldsymbol{p}}{|\boldsymbol{p}|}, l\right) \\
\bar{\Phi}_{m, s}\left(p_{3}, \alpha, \beta, l\right) \\
\bar{\Phi}_{m, s}(\xi, \eta, \alpha, l)
\end{array}\right.
$$


Somit folgt

$$
\left(\Phi,\left(1-E_{0}\right) E(N) \Phi\right)=\int \sum_{s}\left(\Phi_{m, s}, E_{m, s}(N) \Phi_{m, s}\right)_{m, s} d \varrho\left(m^{2}\right)=0
$$

womit die Behauptung bewiesen ist.

Bemerkung. Aus dem Verlauf des Beweises geht hervor, daß es wesentlich ist, daß in den irreduziblen Darstellungen von $\mathscr{G}$ eine ,,hinreichend glatte Verteilung" von irreduziblen Darstellungen der Translationsgruppe $\{\lambda e\}$ vorkommt. Das dürfte immer der Fall sein, falls $\mathscr{G}$ das semidirekte Produkt einer nichtkompakten endlich-dimensionalen abelschen Liegruppe mit einer der klassischen Gruppen ist. Andererseits versagt der Beweis, wenn man z. B. die Translationsgruppe für sich alleine behandelt; denn dann sind die irreduziblen Darstellungen eindimensional, führen also zu einem diskreten Spektralmaß.

Den Herren S. Schlieder und H. ReeH danke ich für den Hinweis auf das behandelte Problem und viele für mich sehr lehrreiche Diskussionen.

\section{Literatur}

1. HaAg, R.: Phys. Rev. 112, 669 (1958); Ruelle, D.: Helv. Phys. Acta 35, 147 (1962); Hepp, K.: Commun. Math. Phys. 1, 95 (1965).

2. Doplioher, S., R. V. Kadison, D. Kastler, and D. W. Robinson: Commun. Math. Phys. 6, 101 (1967).

3. Neumark, M. A.: Normierte Algebren. Berlin: VEB Deutscher Verlag der Wissenschaften 1959.

D. MAISON

Max-Planck-Institut f. Physik u.

Astrophysik

8000 München 23, Föhringer Ring 6 